UCRL-JC-128021

PREPRINT

\title{
Thomson Scattering from Inertial Confinement Fusion Plasmas
}

\author{
S. H. Glenzer, C. A. Back, L. J. Suter, S. M. Pollaine, B. J. MacGowan, \\ M. A. Blain, J. S. De Groot, K. G. Estabrook, B. A. Hammel, \\ J. H. Hammer, O. L. Landen, J. D. Lindl, B. H. Wilde
}

This paper was prepared for submittal to the 13th International Conference on Laser Interactions and Related Plasma Phenomena

Monterey, CA

April 13-18, 1997

July 8, 1997

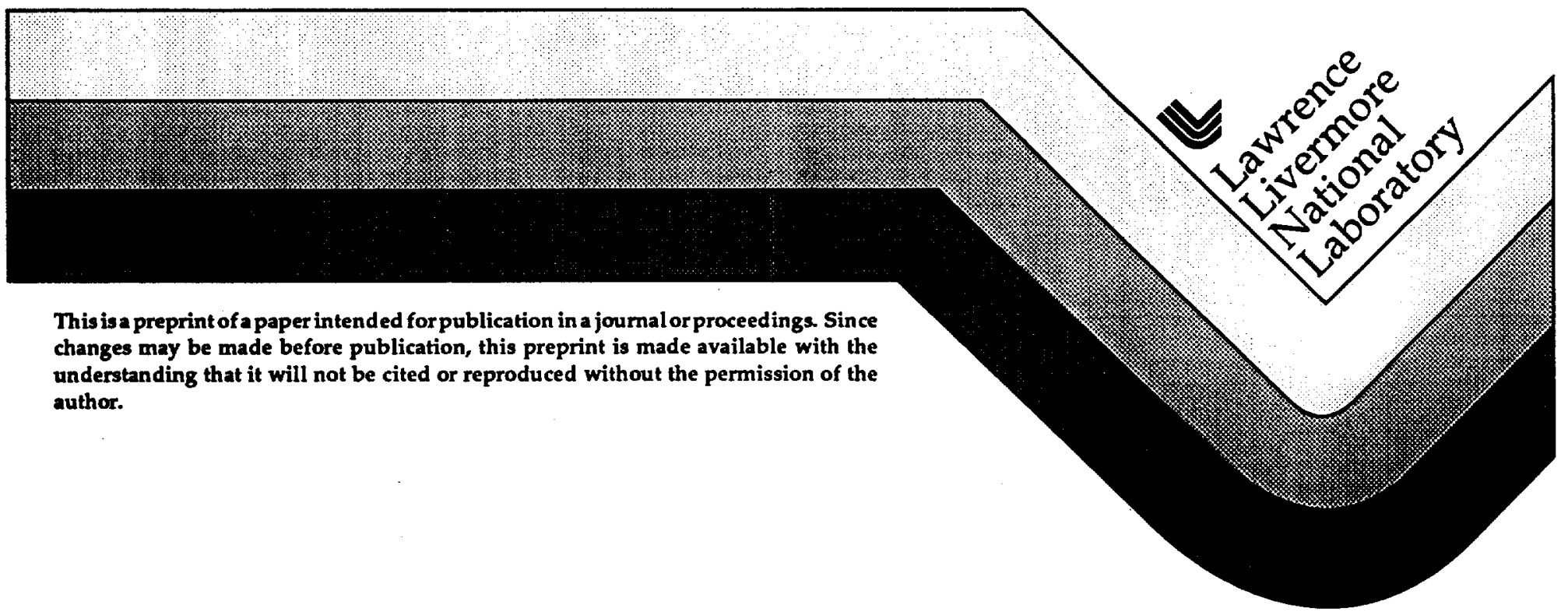




\section{DISCLAMER}

This document was prepared as an account of work sponsored by an agency of the United States Government. Neither the United States Government nor the University of California nor any of their employees, makes any warranty, express or implied, or assumes any legal liability or responsibility for the accuracy, completeness, or usefulness of any information, apparatus, product, or process disclosed, or represents that its use would not infringe privately owned rights. Reference herein to any specific commercial product, process, or service by trade name, trademark, manufacturer, or otherwise, does not necessarily constitute or imply its endorsement, recommendation, or favoring by the United States Government or the University of California. The views and opinions of authors expressed herein do not necessarily state or reflect those of the United States Government or the University of California, and shall not be used for advertising or product endorsement purposes. 


\title{
Thomson Scattering from Inertial Confinement Fusion Plasmas
}

\author{
S. H. Glenzer, C. A. Back, L. J. Suter, S. M. Pollaine, B. J. MacGowan, \\ M. A. Blain*, J. S. De Groot**, K. G. Estabrook, B. A. Hammel, \\ J. H. Hammer, O. L. Landen, J. D. Lindl, and B. H. Wilde*** \\ Lawrence Livermore National Laboratory, P.O. Box 808, L-399, Livermore CA 94550 \\ * Centre D'Etudes de Limeil-Valenton, France \\ ** Department of Applied Science and Plasma Research Group. Davis, CA 95616, USA \\ *** Los Alamos National Laboratory, Los Alamos, New Mexico 87545
}

\begin{abstract}
Thomson scattering has been developed at the Nova laser facility as a direct and accurate diagnostic to characterize inertial confinement fusion plasmas. Flat disks coated with thin multilayers of gold and beryllium were irradiated with one laser beam to produce a two ion species plasma with a controlled amount of both species. Thomson scattering spectra from these plasmas showed two ion acoustic waves belonging to gold and beryllium. The phase velocities of the ion acoustic waves are shown to be a sensitive function of the relative concentrations of the two ion species and are in good agreement with theoretical calculations. These open geometry experiments further show that an accurate measurement of the ion temperature can be derived from the relative damping of the two ion acoustic waves. Subsequent Thomson scattering measurements from methane-filled, ignition-relevant hohlraums apply the theory for two ion species plasmas to obtain the electron and ion temperatures with high accuracy. The experimental data provide a benchmark for twodimensional hydrodynamic simulations using LASNEX, which is presently in use to predict the performance of future megajoule laser-driven hohlraums of the National Ignition Facility (NIF). The data are consistent with modeling using significantly inhibited heat transport at the peak of the drive. Applied to NIF targets, this flux limitation has little effect on x-ray production. The spatial distribution of $x$-rays is slightly modified but optimal symmetry can be re-established by small changes in power balance or pointing. Furthermore, we find that stagnating plasma regions on the hohlraum axis are well described by the calculations. This result implies that stagnation in gas-filled hohlraums occurs too late to directly affect the capsule implosion in ignition experiments.
\end{abstract}

\section{INTRODUCTION}

In the indirect drive approach to inertial confinement fusion, gas-filled hohlraums are used as radiation enclosures converting high-power laser energy into a soft $x$-ray radiation field to achieve a symmetric high-convergence capsule implosion (1). The gas fill is utilized to reduce inward motion of the gold wall plasma and to obtain high soft $x$-ray radiation symmetry $(2,3)$. To understand the physics of gas-filled hohlraums such as the energetics, beam deflection or stagnation processes, we have performed collective Thomson scattering measurements (4-8) from the low- $Z$ gas plasma. These experiments provide temporally and spatially resolved measurements of the electron temperature $T_{e}$, ion 
temperature $T_{i}$ and plasma flow $v$ in ignition-relevant hohlraums (9-11), thus benchmarking hydrodynamic simulations.

The hohlraum plasmas were produced at the Nova laser facility using standard millimeter-size gold cavities filled with methane. A gas fill giving a two ion species plasma including hydrogen increases ion Landau damping of the ion acoustic plasma waves to reduce laser energy losses by stimulated Brillouin scattering (1215). It is therefore adopted in designs of future hohlraums of the National Ignition Facility (NIF) which use a cryogenic hydrogen-helium fill. For these reasons, we performed two independent Thomson scattering experiments to ensure accurate characterization of indirectly driven inertial confinement fusion plasmas.

We first performed an open geometry experiment (16) testing the theory developed by Fejer $(17,18)$ for Thomson scattering spectra from two ion species plasmas. Our experimental Thomson scattering spectra from plasmas produced by irradiating flat disks, which were coated with thin $\mathrm{Au}$ and Be multilayers, clearly show two ion acoustic waves belonging to both species, $\mathrm{Au}$ and $\mathrm{Be}$. The measured phase velocities are in good agreement with the theoretical predictions calculated for the relative concentrations of the two ion species. Besides the measurement of the electron temperature, these studies show that an accurate measurement of the ion temperature can be derived from the relative damping of the two ion acoustic waves. Moreover, plasma flow can be inferred from the Doppler shift of the whole scattering spectra and the relative ion densities can be measured with high accuracy if the ion charge state is known independently or vice versa.

Subsequent Thomson scattering experiments to characterize methane-filled, millimeter-size hohlraums (19) provide a benchmark for two-dimensional hydrodynamic simulations using LASNEX $(11,20)$. This code is presently in use to predict the performance of centimeter-size, megajoule laser-driven hohlraums of the NIF. The measurements show a steep rise of the electron temperature to $5 \mathrm{keV}$ at the peak of the drive which is consistent with calculations only by strongly inhibiting the electron heat transport late in the pulse. A temperature of $\mathrm{T}_{e}=5 \mathrm{keV}$ can be approximated with a flux limiter of $f=0.01$ or equivalently reducing Spitzer conductivity by a factor of 10 . While this finding is not presently understood, it does indicate that it is necessary to include heat transport limiting effects into the modeling, e.g., magnetic fields $(21,22)$ or non local transport $(23)$. In this paper we will present some simulations which include the toroidal magnetic field while neglecting the axial component. These simulations show marginal improvements. Three-dimensional hydrodynamic modeling with the inclusion of magnetic fields as well as experiments quantifying magnetic fields in hohlraums will be necessary to further evaluate the importance of magnetic fields on transport in gas-filled hohlraums. LASNEX simulations of NIF hohlraums show that the symmetry of the fusion capsule is slightly affected by the details. of the heat transport model. Although a heat transport flux limiter of $f=0.01$ does not affect the radiation temperature in a NIF hohlraum, the $\mathrm{x}$-ray radiation flux as a function of the incident angle on the fusion capsule differs somewhat from the standard calculations using $f=0.05$. Although small changes to the beam power balance and pointing can be used to re-optimize symmetry, an improved understanding of heat transport in hohlraums will permit a better specification of the range of suitable ignition hohlraum designs..

The experimental data further show that ion temperatures and plasma flow, i.e., quantities which are not sensitive to the choice of the heat transport flux limiter, agree quite well with the simulations. In particular, the ion temperature measurements at the peak of the drive show a fast rise to $4 \mathrm{keV}$ due to stagnation of 
the compressed plasma on the axis of the hohlraum. The experimental observations are well described by the simulations verifying that high gas pressures from stagnating plasma regions occur late, after the capsule has generated a large surrounding region of ablated material. Our data imply that there is virtually no affect of the gas-region pressure on the capsule implosion in current designs for gas-filled hohlraums.

\section{EXPERIMENTS}

The experiments were performed with the Nova laser facility at the Lawrence Livermore National Laboratory (24). It is a Nd:glass laser operating at $1.055 \mu \mathrm{m}$ $(1 \omega)$ which can be frequency converted to $2 \omega$ or $3 \omega$. To produce a two-ion species plasma with a controlled amount of a light and a heavy species, a flat disk of $2 \mathrm{~mm}$ diameter was illuminated with a single Nova beam. It is a f/4.3 laser beam illuminating the disk at an angle of $64^{\circ}$ to normal. We used a 1 ns square pulse and $2.9 \mathrm{~kJ}$ energy at $3 \omega(\lambda=351 \mathrm{~nm})$. A diverging focus resulted in an intensity of $I=10^{15} \mathrm{~W} \mathrm{~cm}^{-2}$ on target. The spot size was measured to be $350 \times 800 \mu \mathrm{m}^{2}$ by two-dimensional plasma $x$-ray imaging with a temporal resolution of 80 ps. The disks were coated with $\mathrm{Au}$ and $\mathrm{Be}$ multilayers of varying thickness. For example, we used 860 layers of $0.5 \mathrm{~nm} \mathrm{Au}$ and $5.6 \mathrm{~nm}$ Be with a total thickness of $2.6 \mu \mathrm{m}$ on a $51 \mu \mathrm{m}$ thick Au or $254 \mu \mathrm{m}$ thick Be substrate to obtain a plasma consisting of $4 \% \mathrm{Au}$ and $96 \% \mathrm{Be}$.

The hohlraums were heated with eight or nine $3 \omega$ beams with energies of 21-25 kJ. They were cylindrical gold enclosures of $2750 \mu \mathrm{m}$ length and with a radius of $800 \mu \mathrm{m}$. These targets are commonly referred to as scale-1 hohlraums which are standard targets for capsule implosions (9). The gas fill was 1 atmosphere of methane $\left(\mathrm{CH}_{4}\right)$ giving an initial electron density of $n_{e}=2.7 \times 10^{20}$ $\mathrm{cm}^{-3}$ when fully ionized. The hohlraums were heated with 8 or 9 unsmoothed heater beams of the Nova laser that are arranged in cones on either side of the hohlraum so that each beam forms an angle of $50^{\circ}$ to the hohlraum axis. The heater beams penetrate the hohlraum at both ends through laser entrance holes (LEH) of $600 \mu \mathrm{m}$ radius (Fig. 1) which are covered with $0.35 \mu \mathrm{m}$ thick polyimide membranes. The beams cross at the center of the holes and are diverging so that they produce an elliptical spot on the hohlraum wall of about $700 \mu \mathrm{m} \times 500 \mu \mathrm{m}$ size. We applied shaped laser pulses of 2.2 ns duration which rise from $0.6-1.8$ TW per beam within approximately $1 \mathrm{~ns}$ (cf. Fig. 1).

The probe beam used for Thomson scattering was one of the Nova beams operating at $2 \omega\left(\lambda_{i}=526.6 \mathrm{~nm}\right)$ in a $4 \mathrm{~ns}$ long square pulse. For the disk experiments, a laser energy of $100 \mathrm{~J}$ was chosen resulting in an intensity of $\mathrm{I}=8 \mathrm{x}$ $10^{13} \mathrm{~W} \mathrm{~cm}^{-2}$. In the hohlraum experiments, typical energies of $500 \mathrm{~J}<\mathrm{E}<1 \mathrm{~kJ}$ were employed with three different focal spot sizes at the scattering volume. Intensities of the probe laser at the scattering volume are smaller than the nominal intensity because of absorption of the probe. The experiments show no significant effect of the probe laser on the plasma conditions. Especially the hohlraums were diagnosed with probe laser intensities which vary by one order of magnitude without affecting the temperature of the plasma.

The scattered light was imaged at a scattering angle of $\theta=104^{\circ}$ with $\mathrm{f} / 10$ optics and a 1:1.5 magnification onto the entrance slit of a $1 \mathrm{~m}$ spectrometer (Spex, model 1704). An optical streak camera (S-20) was used to record spectra with 30 ps 
temporal resolution. High spatial discrimination of $133 \mu \mathrm{m}$ in the vertical direction and $66 \mu \mathrm{m}$ in the axial direction was obtained by choosing the entrance slit width of the spectrometer to be $200 \mu \mathrm{m}$ and by employing a streak camera slit height of 100 $\mu \mathrm{m}$. The spectrometer employed a $2,400 \mathrm{~g} / \mathrm{mm}$ grating blazed at $500 \mathrm{~nm}$ resulting in a reciprocal linear dispersion of $0.220 \mathrm{~nm} / \mathrm{mm}$ (first order). The wavelength resolution of the measurements was $0.1 \mathrm{~nm}$. Wavelength calibration and absolute calibration of the detection system were performed with a Ne spectral lamp and a tungsten lamp.
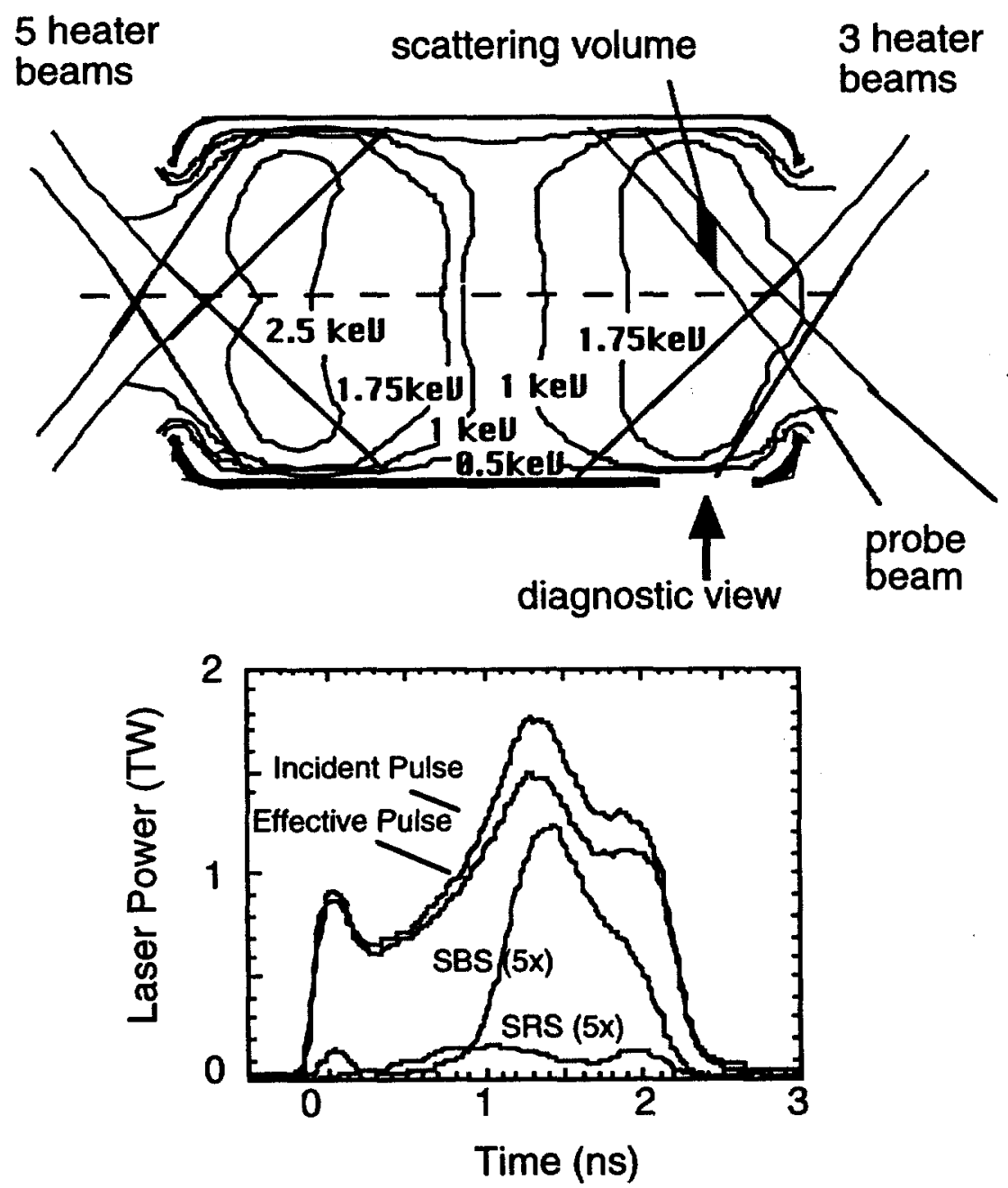

Figure 1. Schematic of the hohlraum setup. An electron temperature contour calculated by LASNEX for a gas-filled hohlraum without a capsule is included for $t=t_{0}+0.8 \mathrm{~ns}$. The location of the scattering volume indicated by the shaded area is in the homogeneous part of the CH-plasma. Also shown is the laser power of a single beam together with measured losses by stimulated Brillouin scattering (SBS) and stimulated Raman scattering (SRS). The total energy loss is $14 \%$.

Collective Thomson scattering is expected for the parameters of the experiments (electron density, temperature, scattering angle, and probe laser wavelength). Typical scattering parameters are $\alpha=1 / k \lambda_{\mathrm{D}}>3$, and light is predominantly scattered 
into the narrow ion feature of the Thomson scattering spectrum (25). In this regime, the spectrum of a single species plasma shows two ion acoustic features (red and blue shift for co- and counter-propagating ion acoustic waves). The frequency separation of the ion acoustic features is twice the ion acoustic frequency, and the propagation direction of the corresponding ion acoustic waves in the plasma is determined by the scattering vector $\mathbf{k}, \mathbf{k}=\mathbf{k}_{\mathbf{s}}-\mathbf{k}_{\mathbf{j}}$. The incident wave vector is defined by $\mathbf{k}_{\mathbf{i}}=\left(2 \pi / \lambda_{j}\right) * \mathbf{e}_{\mathbf{i}}$, where $\lambda_{\mathbf{i}}$ is the incident probe laser wavelength and $\mathbf{e}_{\mathbf{j}}$ is the unit vector in direction of the probe laser. Similarly $\mathbf{k}_{\mathbf{s}}$ points in the direction of the detector. For a two-species plasma with two ion acoustic waves (each with red and blue shift), the Thomson scattering will show four ion acoustic features.

\section{EXPERIMENTAL RESULTS AND DISCUSSION}

\section{Disk Experiments}

Figure 2 shows the Thomson scattering data recorded from a $4 \% \mathrm{Au}, 96 \% \mathrm{Be}$ disk target with the probe laser focused at a distance of $\mathrm{z}=500 \mu \mathrm{m}$ from the disk surface. The probe laser was parallel to the disk and focused to $200 \mu \mathrm{m}$ diameter. Four ion acoustic features, the two outer ones belonging to $\mathrm{Be}$ and the two inner ones belonging to $\mathrm{Au}$, can be clearly seen for $\mathrm{t}_{0}+0.9 \mathrm{~ns}<\mathrm{t}<\mathrm{t}_{0}+1.7 \mathrm{~ns}$. The $3 \omega$ heater beam lasts from 0 to $1 \mathrm{~ns}$ and the $2 \omega$ probe beam from 0 to $4 \mathrm{~ns}$. The Thomson scattering signal is fairly symmetric and starts at $t=t_{0}+0.8 \mathrm{~ns}$. It shows an increasing separation of the ion acoustic features for about 0.2 ns indicating increasing temperatures of the plasma during the heating period. After the end of the heating at $t=t_{0}+1 \mathrm{~ns}$ the separation of the ion acoustic features decreases rapidly since the plasma cools due to radiative cooling and expansion. For the time interval shown in Fig. 2, Thomson scattering gives temperatures of the plasma of $200 \mathrm{eV}<$ $\mathrm{T}_{\mathrm{e}}<900 \mathrm{eV}$ ensuring that the Be ions are fully ionized. The separation and damping of the ion acoustic features belonging to the Be ions can be directly used to determine the electron temperature. In addition, electron densities with an uncertainty of about a factor of two are obtained from the calibration of the detector. This predicts a scattering parameter $\alpha$ well above 3 for our conditions, in which case scattering spectra are not sensitive to the electron density.

The signal detected about $0.8 \mathrm{~ns}$ before the onset of the Thomson scattering signal is due to unconverted $2 \omega$ stray light from the heater beam. This feature is not caused by the probe beam and represents a convenient timing and wavelength fiducial. The delay of $0.8 \mathrm{~ns}$ is due to the time needed for ablation and travel of the plasma from the disk surface to the scattering volume. When the plasma reaches the scattering volume it is moving towards the observer resulting in a blue shift of the Thomson scattering signal. The shift yields the instantaneous macroscopic plasma motion along the scattering vector $k$. The measured blue shift of $1.7 \mathrm{~nm}$ at $t=t_{0}+$ $0.9 \mathrm{~ns}$ corresponds to a velocity of $10^{8} \mathrm{~cm} / \mathrm{s}$. It is obvious from Fig. 2 that this is well above the sound speed since the shift of the whole Thomson scattering signal is larger than half the wavelength separation of the ion acoustic features.

Figure 2 (b) shows a spectrum at $t_{0}+1.5 \mathrm{~ns}<\mathrm{t}<\mathrm{t}_{0}+1.6 \mathrm{~ns}$. Four ion acoustic features due to the slow and the fast ion acoustic waves are clearly identified. The small asymmetry is probably caused by the heat flux driven return current (26). The experimental data are fitted with a theoretical spectrum which compensates for the 
asymmetry by slightly decreasing the damping of the waves on the red wing $(6,27)$. Apart from the slight asymmetry, analysis of the spectrum yields considerable information. Since the relative intensities of the ion acoustic features are determined by damping of the $\mathrm{Au}$ and $\mathrm{Be}$ ion acoustic waves, the theoretical fit to the spectrum gives $\mathrm{T}_{\mathrm{i}} / \mathrm{T}_{\mathrm{e}}=1.1$ (Fig. 2(c)). From the wavelength separation of the $\mathrm{Be}$ ion acoustic features, we find $\mathrm{T}_{\mathrm{e}}=230 \mathrm{eV}$. We can then infer $\alpha=7$ for the electron densities from the intensity of the scattering signal and from hydrodynamic simulations. Finally, the wavelength separation of the Au features gives $Z=40$. As can be seen, the theoretical spectrum fits the experimental data quite well. Small deviations are probably due to small plasma parameter gradients within the scattering volume.

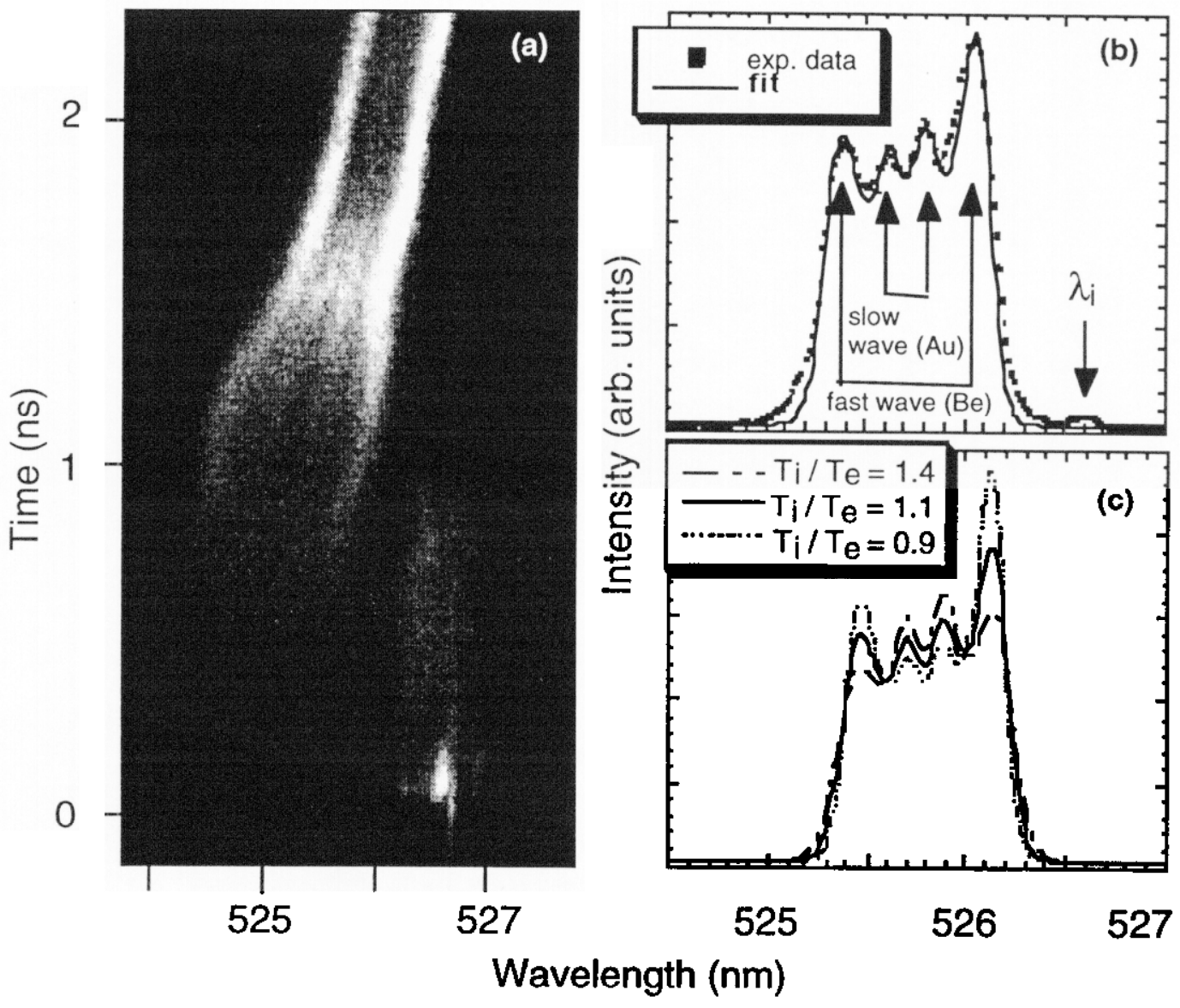

Figure 2. Time-resolved Thomson scattering spectrum from a $4 \% \mathrm{Au}, 96 \%$ Be plasma detected at a distance of $500 \mu \mathrm{m}$ from the target (a). Also shown is the Thomson scattering spectrum at $t=t_{0}+1.55$ ns with a theoretical fit (b), and the variation of the scattering spectrum for various ratios of $T_{i} / T_{\theta}$ (c).

Figure 3 shows the compilation of our experiments with relative $\mathrm{Au}$ concentrations of $1 \%, 2 \%, 4 \%$, and $8 \%$ for experiments using an unsmoothed Nova heater beam and for experiments using a kinoform phase plate in heater beam. The ratio of the wavelength separation of the $\mathrm{Au}$ ion acoustic features to the $\mathrm{Be}$ ion acoustic features is plotted as a function of the Au concentration. 
These data are taken at $t=t_{0}+1.55 \mathrm{~ns}$ for the unsmoothed case and at at $t=t_{0}+$ $1.3 \mathrm{~ns}$ for the smoothed case when the intensity of the Au features is at its maximum. For very small Au concentrations of $1 \%$, the Thomson scattering signal shows a central peak belonging to the heavy $\mathrm{Au}$ ions in addition to the ion acoustic waves belonging to the $\mathrm{Be}$ ions. The existence of a central peak was also observed in a two-species discharge plasma consisting of hydrogen and small amounts of heavy gases $(27,28)$. Increasing the amount of the Au species of the two-species plasma results in a larger separation of the ion acoustic features of Au. For the 2\% case the ion acoustic waves of Au become discernible as two individual peaks on the spectrum. For a mixture of $4 \% \mathrm{Au}, 96 \%$ Be two separate ion acoustic waves were clearly observed (Fig. 2). By further increasing the amount of $\mathrm{Au}$ to $8 \%$ the ion acoustic waves of $\mathrm{Au}$ and $\mathrm{Be}$ almost merge together. From the theoretical fit of the spectra we find that $T_{i} / T_{e}$, the scattering parameter $\alpha$, and charge state $Z$ of the $\mathrm{Au}$ ions are the same for all experiments to within $15 \%$.

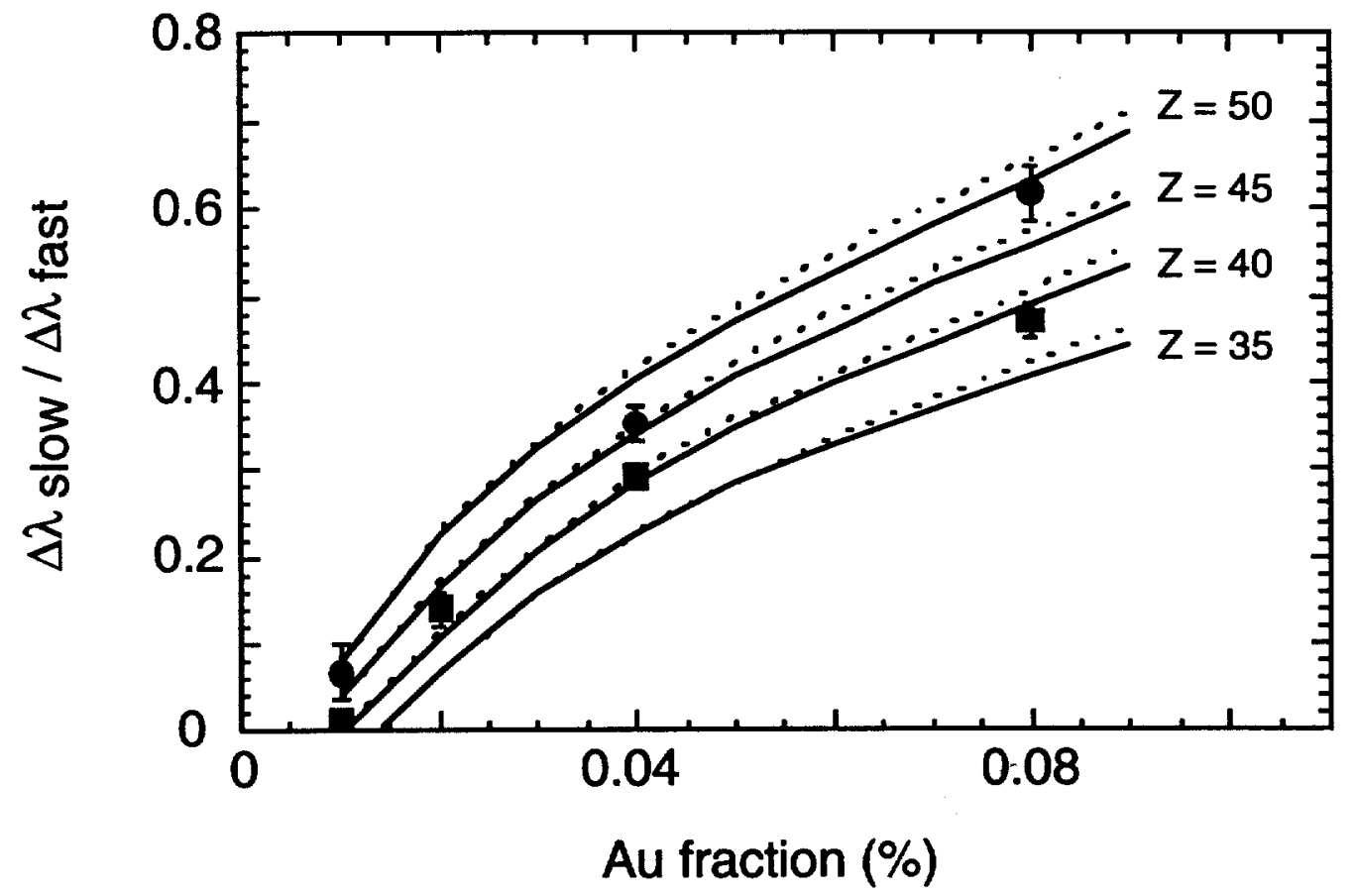

Figure 3. Ratio of the wavelength separations of the ion acoustic features belonging to $\mathrm{Au}$ to those belonging to $\mathrm{Be}$ on the Thomson scattering signal for different $\mathrm{Au}$ concentrations. Ratios are shown for unsmoothed heater beams (full squares) which were taken at $t=t_{0}+1.55$ ns when $T_{i} / T_{e} \sim 1.1$ and $\alpha \sim 7$. Also plotted are experimental ratios with a kinoform phase plate in the heater beam taken at $t=t_{0}+1.3 \mathrm{~ns}$ when electron temperatures and $Z$ are slightly larger giving $T_{i} / T_{e} \sim 1.3(0)$ The error bars are estimated from the noise of the data. Theoretical data after Evans (18) are shown for $Z=35,40,45$, $50, T_{i} / T_{e} \sim 1.1$ (full line) and $T_{i} / T_{e} \sim 1.3$ (dots).

The experimental data clearly show the increase of the wavelength separation of the $\mathrm{Au}$ ion acoustic feature with increasing concentration. The theoretically calculated results (18) are also plotted for various $\mathrm{Z}$ of the $\mathrm{Au}$ ions. The experimental data are in excellent agreement with the theory for $Z=40$ for the 
unsmoothed case and for $Z=45$ for the smoothed case. The latter resulted in slightly larger temperatures and consequently slightly larger ionization stages. The values of $Z$ compares quite well with hydrodynamic LASNEX calculations which give $Z=45$ and $Z=49$, respectively.

Our experimental results are in excellent agreement with the theory of Fejer (17) and Evans (18). Our data show that Thomson scattering from a two ion species plasma is an accurate diagnostic of electron temperature and ion temperature. The observation of both the fast and the slow wave on the Thomson scattering spectra shows that they are moderately to strongly damped. In this regime, Landau damping is a strong function of the ion temperature resulting in a highly accurate ion temperature measurement. In addition, the relative ion densities can be measured with high accuracy if the ion charge state is known independently or vice versa.

\section{Hohlraum Experiments}

Figure 4 shows the Thomson scattering data from a gas-filled hohlraum heated with 8 heater beams with a total energy of $21 \mathrm{~kJ}$.
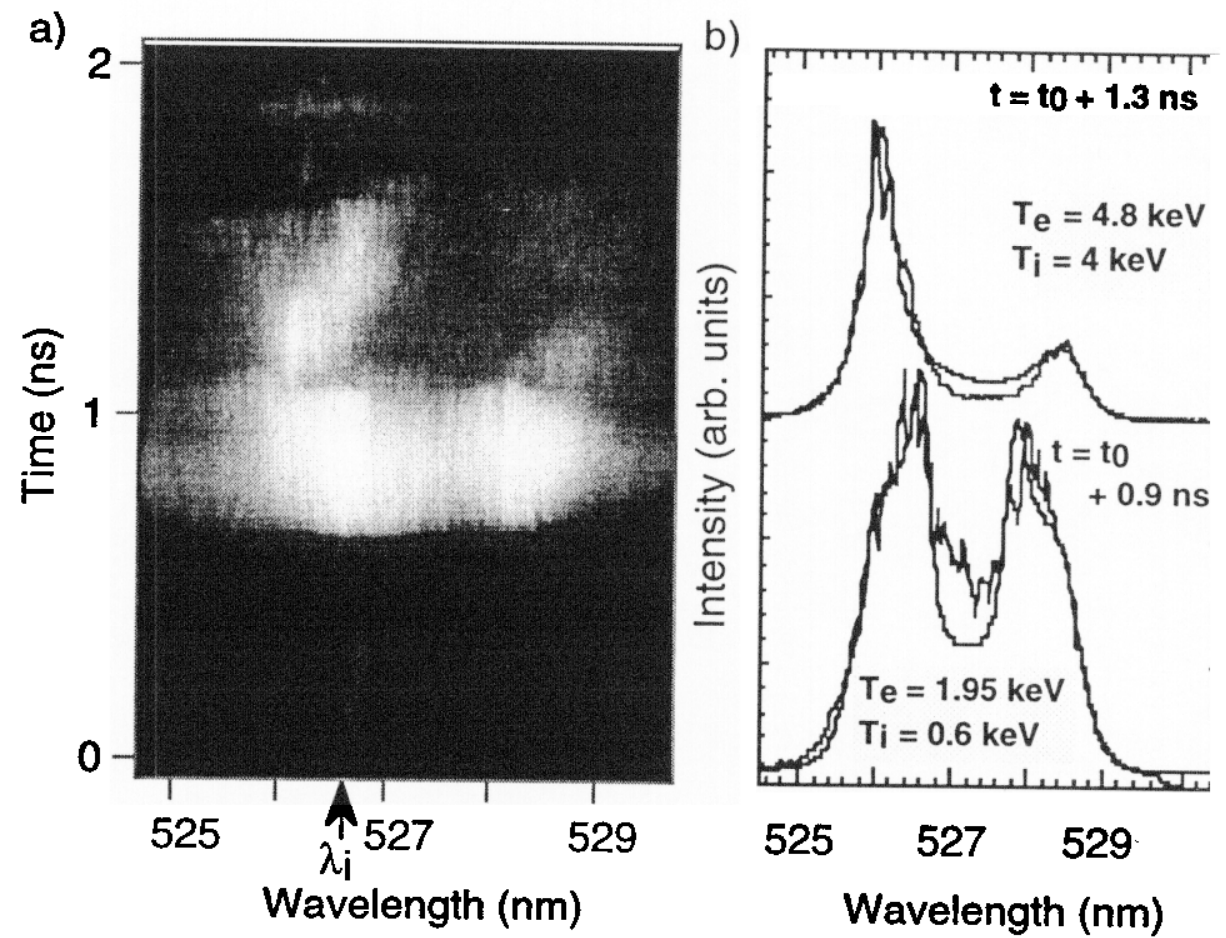

Figure 4. Time-resolved Thomson scattering spectrum from $\mathrm{CH}_{4}$ for 8 heater beams (a) inside the hohlraum. The spectra are fitted with the form factor of Evans (18) giving electron and ion temperature of the plasma (b).

The scattering volume is $400 \mu \mathrm{m}$ inside of the hohlraum (cf. Fig. 1) and the Thomson scattered light is observed through a diagnostic window $(700 \mu \mathrm{m} x$ $400 \mu \mathrm{m}$ ) which is cut into the hohlraum wall and also covered with polyimide. For 
$\mathrm{t}_{0}<\mathrm{t}<\mathrm{t}_{0}+0.65$ ns no Thomson scattering signal can be observed. Estimates show that during this time the polyimide foil which covers the diagnostic window produces an overdense plasma for $2 \omega$ light. During that time a faint line due to stray light or unconverted $2 \omega$ light from the heater beams can be identified on Fig. 4(a). Again, it is used as a timing and wavelength fiducial. For $\mathrm{t}_{0}+0.65 \mathrm{~ns}<\mathrm{t}<\mathrm{t}_{0}+$ $1.1 \mathrm{~ns}$ two broad symmetric ion acoustic features are observed from light scattering off the $\mathrm{CH}_{4}$-plasma. For $\mathrm{t}_{0}+1.1 \mathrm{~ns}<\mathrm{t}<\mathrm{t}_{0}+1.6 \mathrm{~ns}$, when the heater beam power rises significantly (Fig. 1), the separation of the ion acoustic features increases indicating a rising electron temperature and, in addition, the width of the ion acoustic features narrows showing an even faster increase of the ion temperature of the plasma. Furthermore, the Thomson scattering spectrum shows an asymmetry probably related to electron heat flux towards the hohlraum wall which would result in different electron Landau damping of the co- and counterpropagating ion acoustic waves $(26,29)$. For $t>t_{0}+1.6 \mathrm{~ns}$, a cut-off of the Thomson scattering signal occurs because the electron density of the CH-plasma rises steadily during the heating of the hohlraum
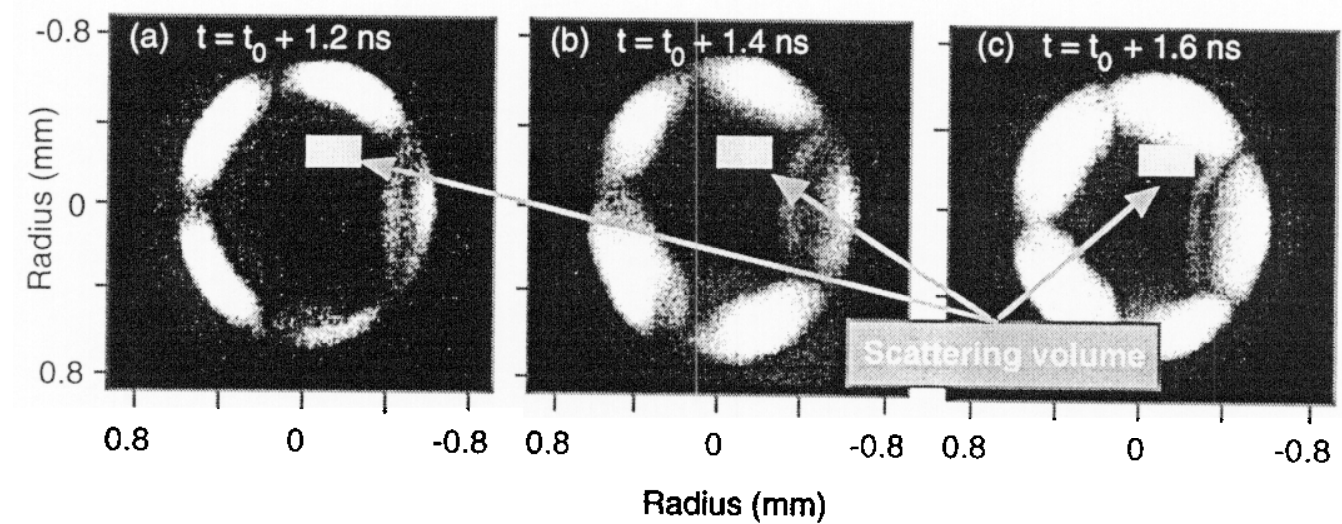

Figure 5. Time-resolved two-dimensional $x$-ray images of the hohlraum gold emission with energies $E>2.5 \mathrm{keV}$ observed along the hohlraum axis.

due to compression by the inward-moving wall plasma. The Thomson scattering spectra from the hohlraum experiments consist of four features: Two co- and counter-propagating ion acoustic waves belonging to $\mathrm{C}$ (slow wave) and to $\mathrm{H}$ (fast wave) $(12,16)$. Fitting the data with the form factor for a two ion species plasma, which has been tested with experiments described above, yields the separation and the relative damping of both waves measuring accurately the electron and ion temperature of the plasma (16).

Figure 4 (b) shows the spectra at $t=t_{0}+0.9$ ns and $t=t_{0}+1.3$ ns averaged over 80 ps together with a fit using the form factor of Evans (18). For the data analysis it is assumed that light scattering occurs on a fully ionized $\mathrm{CH}$-plasma. Our hydrodynamic simulations as well as temporally resolved two-dimensional $\mathrm{x}$-ray images observing the Au-plasma emission at energies of $\mathrm{E}>2.5 \mathrm{keV}$ show that $\mathrm{Au}-$ ions were not present in the scattering volume for $t<t_{0}+1.7$ ns (Fig. 5). The $x-$ ray images view the hohlraum plasma along its axis. They show a five-fold radiation pattern due to the gold blowing off the wall where the heater beams illuminate the hohlraum. Therefore, the radiation of the gold blow-off from the five 
heater beams on either side overlap on the images. At $t=t_{0}+1.6 \mathrm{~ns}$ (Fig. 5(c)), the gold emission close to the scattering volume is therefore not from that side of the hohlraums where we have performed the Thomson scattering measurements and is not affecting the data.

If small amounts of highly ionized gold ions exist in the scattering volume, the Thomson scattering spectra would clearly indicate their presence by showing a central peak. For example, $5 \%$ Au can be readily detected. This is demonstrated in Figure 6 where we compare calculated Thomson scattering spectra of three ion species plasmas. The parameters are $T_{e}=2 \mathrm{keV}, \mathrm{T}_{\mathrm{i}}=0.6 \mathrm{keV}, \mathrm{n}_{\mathrm{e}}=1.3 \times 10^{21}$ $\mathrm{cm}^{-3}$, and assuming a fully ionized methane plasma with various amounts of gold impurities ionized to $Z=50$. For a gold ion density of $N_{A u}=10^{18} \mathrm{~cm}^{-3}$ which corresponds to $0.8 \%$ gold $(\mathrm{Z}=50)$ in a fully ionized methane plasma, the Thomson scattering spectrum deviates only slightly from the case without gold impurities. The assumption of a pure $\mathrm{CH}$-plasma has virtually no affect on the inferred plasma parameters. However, assuming larger gold impurities of $\mathrm{N}_{\mathrm{Au}}=5 \times 10^{18} \mathrm{~cm}^{-3}$ (corresponding to $4.8 \%$ gold in $\mathrm{CH}$ ) significantly enhances the center of the spectrum and the ion acoustic wave which belongs to hydrogen (fast wave) is significantly less compared to the ion acoustic wave belonging to carbon (slower wave).

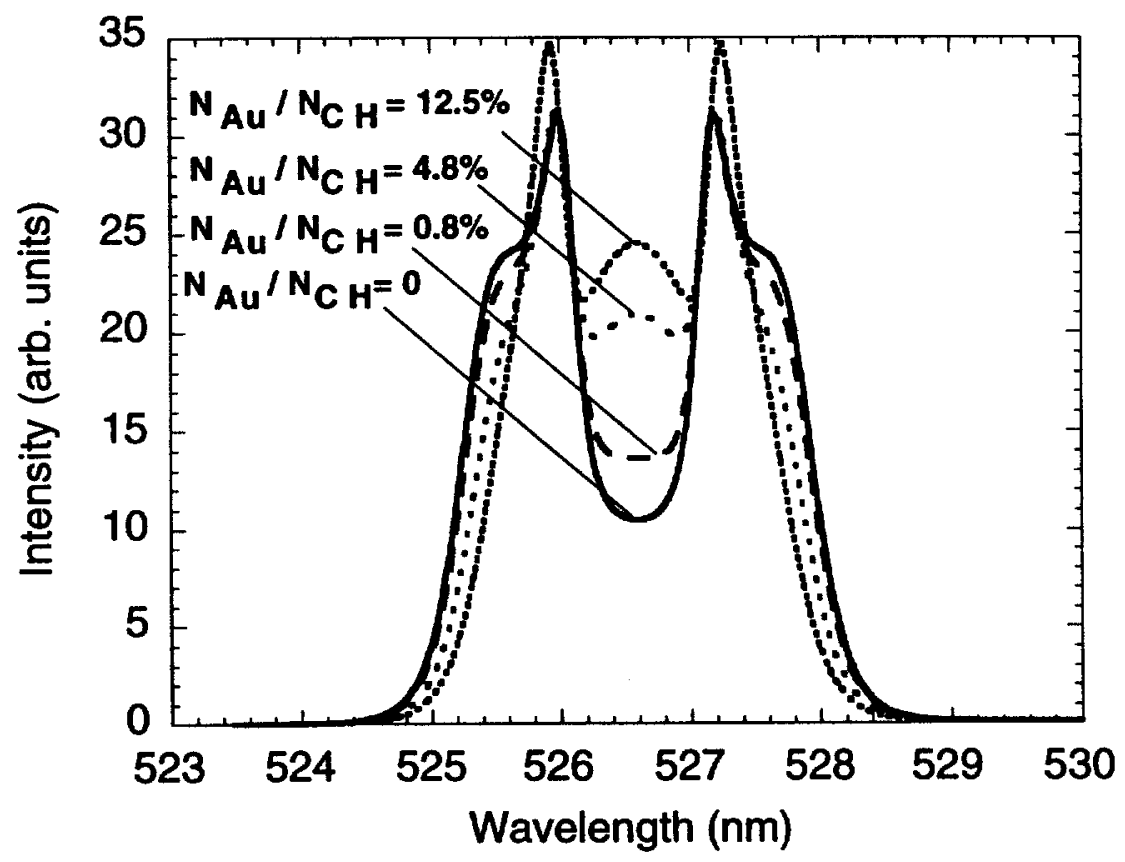

Figure 6. Calculated Thomson scattering spectra for a methane plasma $\left(\mathrm{CH}_{4}\right)$ with various amounts of gold impurities with $Z=50$.

These features become even clearer for $\mathrm{N}_{\mathrm{Au}}=10^{19} \mathrm{~cm}^{-3}$ (corresponding to $12.5 \%$ gold in $\mathrm{CH}$ ). It is obvious that neglecting the gold impurities would result in large errors for the ion temperature (throughout this paper we assume that the temperatures of the ion species are equal because of fast ion-ion equilibration times (30) in the plasmas under study). On the other hand, the Thomson scattering spectrum provides us with a clear indication of the impurity concentration. 
Experimentally we do not observe an enhancement of the center of the Thomson scattering spectrum and conclude that gold impurities are negligible for our conditions (Fig. 4 (b), $\mathrm{t}=\mathrm{t}_{0}+1.3 \mathrm{~ns}$ ).

In Figure 7 we plot the temporal evolution of the electron temperature from Thomson scattering along with the results of the radiation hydrodynamic LASNEX simulations. Data are shown for 8 heater beams (a) and for 9 heater beams (b). The experimental electron temperatures clearly scale with the number of heater beams. We observe peak values of $4.5 \mathrm{keV}$ and $5.2 \mathrm{keV}$, respectively. The reproducibility of the measurements from 12 shots is about $20 \%$.

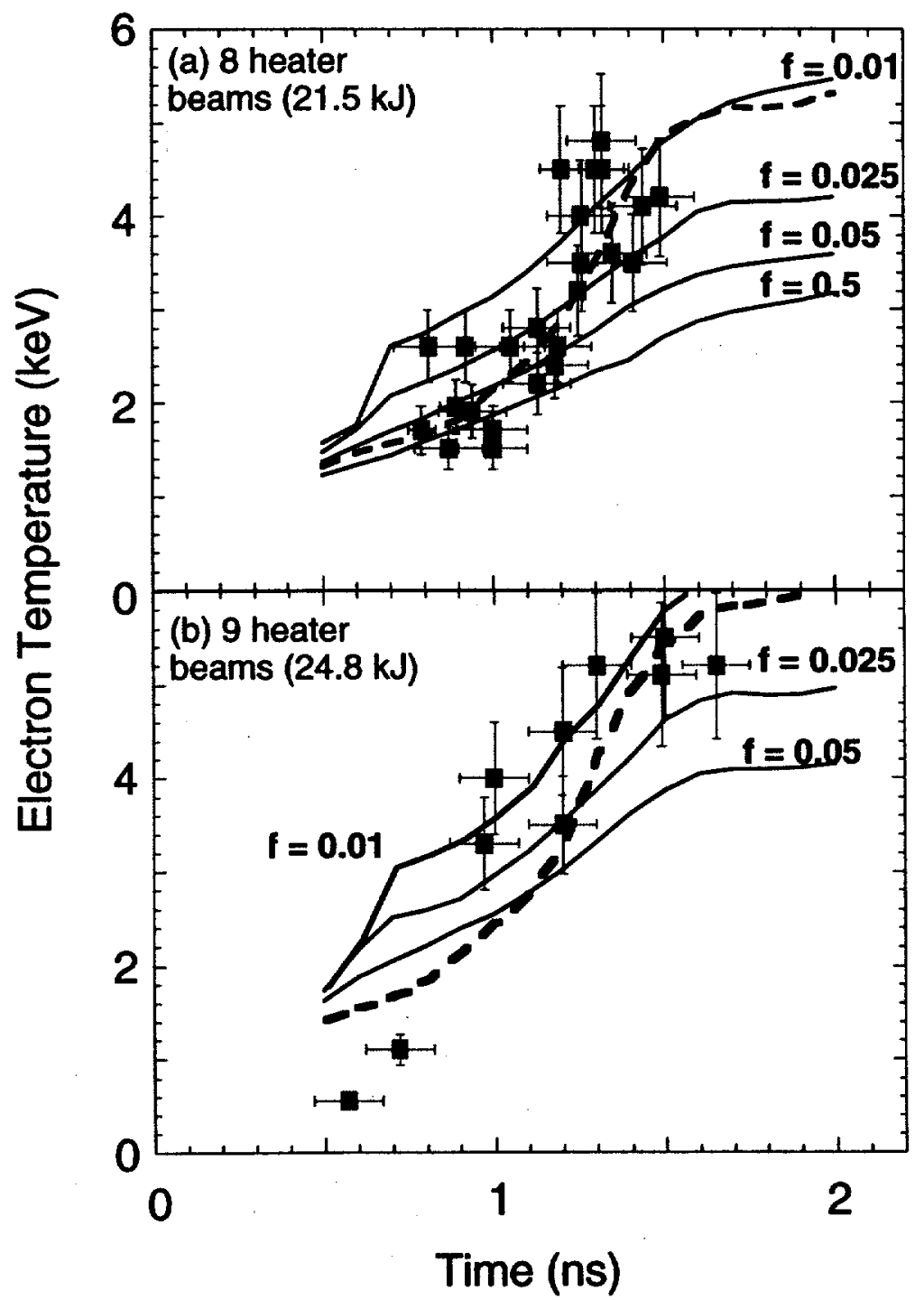

Figure 7. Hohlraum electron temperatures as a function of time for 8 (a) and 9 (b) heater beams together with hydrodynamic simulations with various electron heat flux limiters. The dashed curves employ a time-varying flux limiter and show good agreement with the experimental electron temperatures.

Varying the probe laser focus in the range of 150 to $500 \mu \mathrm{m}$ diameter at the scattering volume did not affect the experimental temperatures. This result is 
due to magnetic fields by a factor of about 20 was predicted by Bodner (21). Also, non local heat transport $(23,34)$ could be important for the present observations. Experiments and calculational efforts (35) are presently ongoing to better understand and quantify these effects.

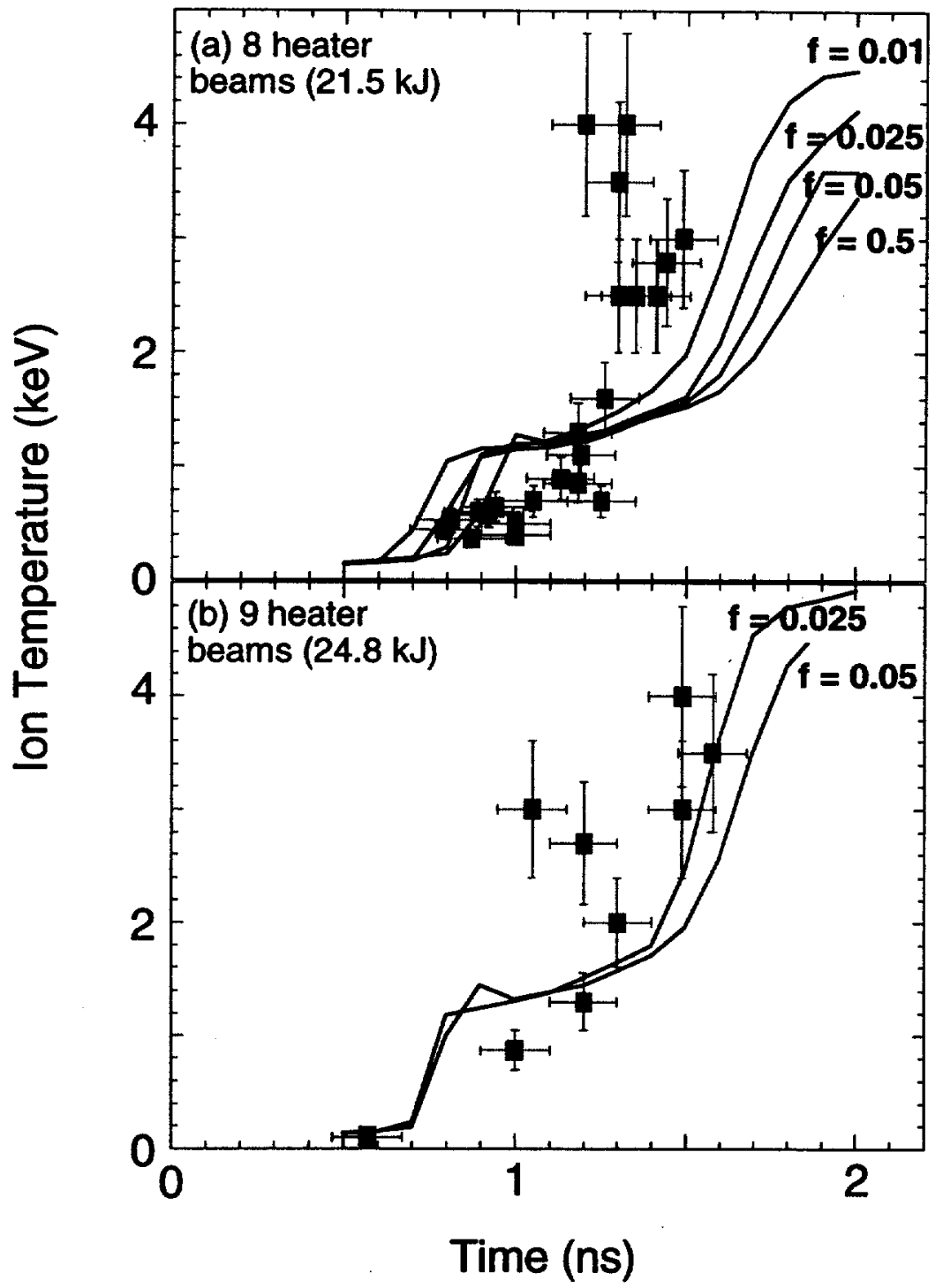

Figure 8. Hohlraum ion temperatures as a function of time for 8 (a) and 9 (b) heater beams together with hydrodynamic simulations with various electron heat flux limiters. The temperature of the hydrogen and carbon ions is assumed to be equal because of high ion ion collision frequencies. Ion temperatures are not sensitive to the choice of the heat flux limiter. They show a steep rise because of stagnation effects.

A variety of calculational tests were performed to verify that besides the electron heat transport model other approximations in the calculation are not important for the present study. An affect on the electron temperature of $<5 \%$ was seen when we varied the focal spot size in the two-dimensional code to test the approximation of 
the laser beam power used in the simulations, or when we included hot electron in the simulations.

Very recently we have performed LASNEX simulations which include a preliminary model for self-consistent toroidal magnetic fields. They use $f=0.5$ which is equivalent to classical heat transport and show an increase of the electron temperature by $7 \%$ at the Thomson scattering volume. This increase is not sufficient to explain our experimental observations. However, the calculations do show localized hot spots near the center of the hohlraum. It is obvious that threedimensional calculations will be necessary to obtain a definitive answer on the importance of magnetic fields on electron heat transport in gas-filled hohlraums.

The Thomson scattering data also give information about macroscopic plasma flow in hohlraums which is an important parameter affecting beam pointing $(32,36)$. In Fig. 2 we observe a red shift of the spectra due to plasma motion away from the heater beam spots on the hohlraum wall. The speed is subsonic $(v=4.3 \mathrm{x}$ $10^{7} \mathrm{~cm} / \mathrm{s}$ ) and underestimated by the two-dimensional simulations by a factor of 23. This Doppler shift (as well as the heat-flux driven asymmetry) is a threedimensional effect and occurs when employing only 8 heater beams. When applying 9 heater beams, the red shift of the spectra decreases to about $20 \%$ of the sound speed $\left(\mathrm{v}=1.4 \times 10^{7} \mathrm{~cm} / \mathrm{s}\right)$ and the spectra become symmetric because of the improved symmetry of the hohlraum heating. In this case, agreement of the flow velocity with the hydrodynamic simulations is obtained within $30 \%$. Unfortunately, the Thomson scattering spectra with 9 heater beams show increased stray light levels since the additional heater beam illuminates part of the diagnostic window.

Ion temperatures as a function of time for 8 or 9 heater beams are shown in Fig. 8. They are less sensitive to the heat flux limiter. The experimental ion temperatures show a steep rise to a peak value of $4 \mathrm{keV}$. From the hydrodynamic simulations we deduce that this behavior is due to stagnation of the compressed low-Z plasma on the axis of the hohlraum. Electron-ion temperature equilibration times are too large ( $>1 \mathrm{~ns}$ ) to explain the experimental data. In Fig. 8 the simulated ion temperature rise is delayed compared to the experimental data by $\sim 0.4 \mathrm{~ns}$ while in case of 9 heater beams a delay of $\sim 0.2 \mathrm{~ns}$ occurs. These observations can be explained by the fact that the hydrodynamic simulations are two-dimensional and assume a cylindrical symmetric heating. However, in the case of 8 heater beams the heating is fairly asymmetric so that the plasma stagnates slightly off-axis closer to the Thomson scattering volume giving rise to an earlier ion temperature increase than seen in the simulations. In case of 9 heater beams the symmetry of the heating is improved and stagnation occurs closer to the hohlraum axis so that the beginning of the ion temperature rise is better described by the LASNEX simulations. In particular, the reasonable agreement between the ion temperatures measured at the peak of the drive and the maximum calculated ion temperatures shows that stagnation processes in gas-filled hohlraums are rather well described by the simulations

\section{Consequences for NIF hohlraums}

When a reduced flux limiter of $f=0.01$ is used in NIF calculations, the optimal beam power balance and the optimal beam pointing is slightly modified because the $x$-ray flux seen by the capsule as a function of angle is modified. This is shown in Fig. 9 where the results of LASNEX simulations are shown for constant flux 
limiters of $f=0.05$, which is the standard flux limiter, and $f=0.01$ to estimate the maximum effect of heat transport inhibition as it is seen in Nova hohlraums on NIF simulations.

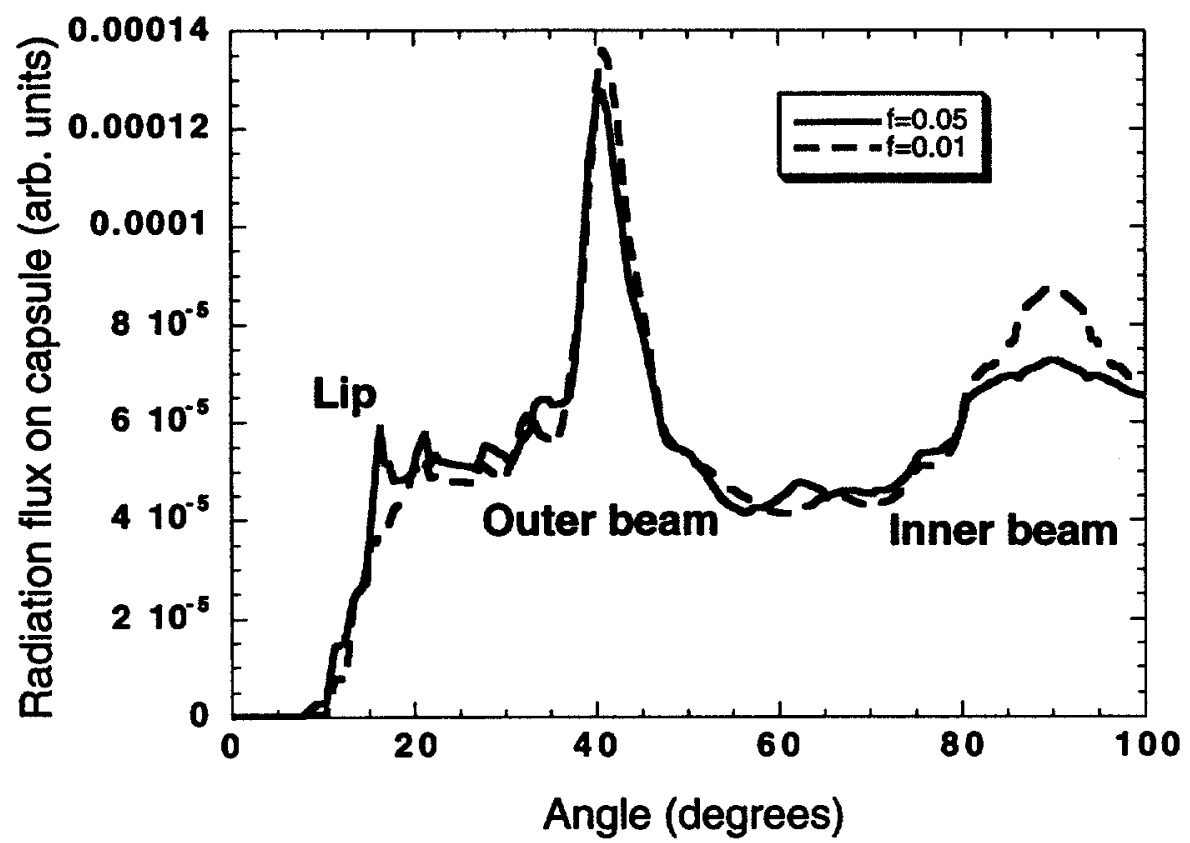

Figure 9. Calculated NIF antenna pattern, i.e. the $x$-ray flux seen by the capsule as a function of angle, at $t=t_{0}+7.5 \mathrm{~ns}$ and for constant flux limiters of $f=0.05$ and $f=0.01$.

These calculations show that increasing the heat transport inhibition by going from $f=0.05$ to $f=0.01$ results in a slightly increased $x$-ray flux from the locations where the inner and outer beams illuminate the hohlraum walls while the $x$-ray radiation from the lip of the laser entrance hole is reduced. The positions of these regions are schematically shown in Fig. 10.

These simulations confirm that strongly inhibiting heat transport in the NIF hohlraum design results in slightly less $\mathrm{x}$-ray production in wall regions which are not directly heated by the laser beams and which are partly heated by conduction. The $x-$ ray flux produces an ablation pressure on the fusion capsule which must be very symmetric to achieve high convergence and ignition of the fusion capsule.

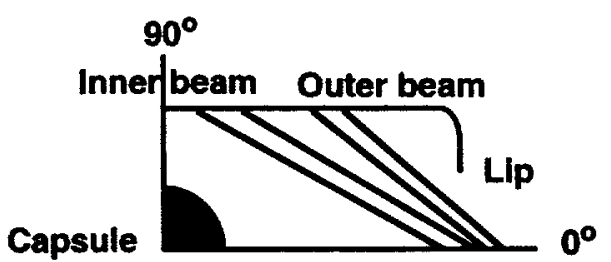

Figure 10. Schematic of the NIF hohlraum showing the locations of high $x$-ray flux production as seen by the capsule.

While the total $\mathrm{x}$-ray flux is not affected by the increased heat transport inhibition (see inset of Fig. 11), the symmetry of the fusion capsule is modified. In Figure 11 we show the ratio P2/P0 as a function of time which has been optimized for $f=$ 0.05 by adjusting beam power and pointing. $\mathrm{P} 2$ is the coefficient of the Legendre 
polynomial describing the pole-equator asymmetry while PO describes the spherical flux. Optimization means reducing the capsule asymmetries by minimizing the ratio P2/P0 (as well as higher order Legendre polynomials).

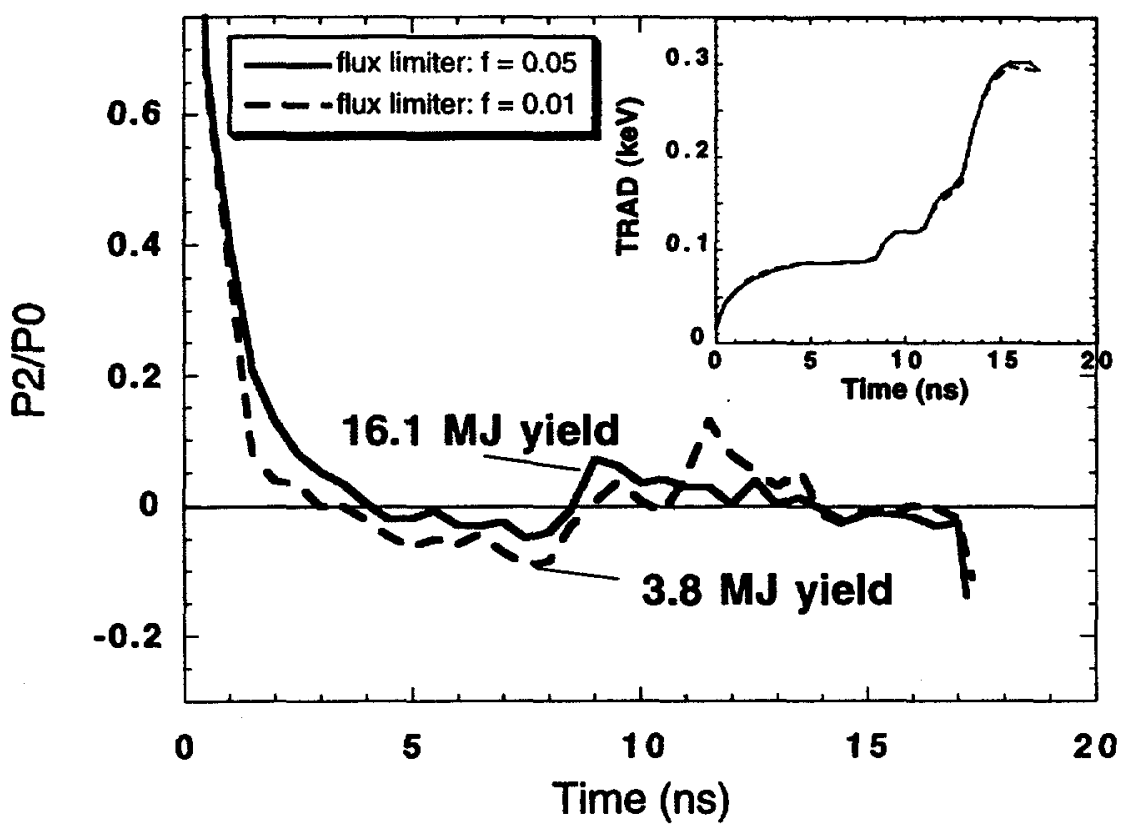

Figure 11. Calculated ratio of the P2/P0 symmetry of the NIF capsule for constant flux limiter of $f=0.05$ and $f=0.01$. The inset shows the radiation temperature as function of time. Both calculations essentially overlap. In case of $f=0.01$ re-optimization of the beam power and pointing will be necessary to increase the capsule gain.

Using beam power and pointing which was optimized for $f=0.05$ for the calculations with $f=0.01$, results in larger amplitudes of the ratio P2/P0 and consequently the capsule yield reduces from $16.1 \mathrm{MJ}$ to $3.8 \mathrm{MJ}$. The pointing and relative power of the beams have to be adjusted in order to improve the capsule yield.

Finally, the reasonable agreement between the experimentally observed stagnation and the LASNEX simulations shows that capsule implosions in gas-filled hohlraums are not directly affected by the pressures produced in the stagnation regions. For example, early NIF hohlraums used plastic liners (1) instead of a gas fill to produce the low $\mathrm{Z}$ beam propagation region in the hohlraum interior. These liners blew inward at high velocity and generated an early time axial stagnation pressure which affected the subsequent capsule implosion symmetry. However, in the gas-filled hohlraums, the high gas pressures occur late, after the capsule has generated a large surrounding region of ablated material. In these hohlraums, there is virtually no affect of the gas-region pressure on the capsule implosion. Because the capsule is moving inward and the radiation ablated capsule blowoff is moving outward at speeds comparable to the sound speed in the gas fill, the capsule is essentially isolated from the pressure generated in the gas. This is true for both the Nova experiments and for the NIF target designs. In NIF calculations, the capsules have essentially the same symmetry and implosion velocity in integrated 
calculations which explicitly account for the low-Z gas pressure $(2,3,11)$ and in separate calculations which only drive the capsule with the hohlraum $x$-ray flux. However, the effect of the stagnation plasma regions on the level of parametric instabilities, which result in e.g. filamentation and backscatter losses by SBS and SRS has not yet been quantified.

\section{CONCLUSIONS}

We have developed Thomson scattering as a powerful diagnostic of inertial confinement fusion plasmas. Open geometry experiments were performed verifying the theory of Fejer $(17,18)$. They show that Thomson scattering measures temporally and spatially resolved electron and ion temperature in two ion species plasmas with high accuracy from the frequency separation and damping of the ion acoustic waves. Furthermore, plasma flow can be inferred from the Doppler shift of the whole scattering spectra and the relative ion densities can be determined if the ion charge state is known independently or vice versa. Applying this theory for the Thomson scattering spectra from closed geometry hohlraum plasmas produced with $21-25 \mathrm{~kJ}$ at the Nova laser facility benchmarked radiation hydrodynamic LASNEX simulations. These studies are important for our understanding of heat transport, energetics and beam pointing in gas-filled hohlraums. The ion temperatures and plasma flow, i.e., quantities which are not sensitive to the choice of the heat transport flux limiter, agree quite well with the simulations. This implies that stagnation effects are well described by LASNEX. The simulations show that stagnation occurs too late in gas-filled hohlraums so that there is no direct effect of the high pressure stagnating plasma on the fusion capsule. On the other hand, the experimental electron temperatures are best approximated with a time-varying flux limiter showing large electron heat transport inhibition at the peak of the drive. This observation is presently not understood. We have started to include heat transport limiting effects into the hydrodynamic modeling but more calculational and experimental work remains to be done for a complete description of the heat transport in gas-filled hohlraums. This study further shows that detailled measurements such as Thomson scattering will be extremely helpful for our understanding of the physics in ignition experiments.

\section{ACKNOWLEDGMENTS}

We thank E. M. Campbell, A. A. Offenberger, A. J. MacKinnon, and D. Hinkel for helpful discussions. This work was performed under the auspices of the U.S. Department of Energy by the Lawrence Livermore National Laboratory under contract No. W-7405-ENG-48.

\section{REFERENCES}

1. J. D. Lindl, Phys. Plasmas 2, 3933 (1995).

2. S. W. Haan, S. M. Pollaine, J. D. Lindl, L. J. Suter, R. L. Berger, L. V. Powers, W. E. Alley, P. A. Amendt, J. A. Futterman, W. K. Levedahl, M. D. Rosen, D. P. Rowley, R. A. Sacks, A. I. Shestakov, G. L. Strobel, M. Tabak, S. V. Weber, G. B. Zimmerman, W. J. 
Krauser, D. C. Wilson, S. V. Coggeshall, D. B. Harris, N. M. Hoffman, and B. H. Wilde, Phys. Plasmas 2, 2480 - 2487 (1995).

3. W. J. Krauser, N. M. Hoffman, D. C. Wilson, B. H. Wilde, W. S. Varnum, D. B. Harris, F. J. Swenson, P. A. Bradley, S. W. Haan, S. M. Pollaine, A. S. Wan, J. C. Moreno, and P. A. Amendt, Phys. Plasmàs 3, 2084 - 2093 (1995).

4. H.-J. Kunze, in! Plasma Diagnostics, edited by W. Lochte-Holtgreven (North-Holland, Amsterdam, 1968) p.550.

5. A. W. DeSilva and G. C. Goldenbaum, in Methods of Experimental Physics, edited by H. R. Griem and R. Lovberg (Academic, New York, 1967), Sec. V9A.

6. D. E. Evans and Katzenstein, Rep. Pro. Phys. 32, 207 - 271 (1969).

7. J. Sheffield, Plasma Scattering of Electromagnetic Radiation (Academic, New York, 1975).

8. N. C. Luhman, Jr. and W. A. Peebles, in Laser Handbook, edited by M. Bass and M. L. Stitch (North-Holland, Amsterdam, 1985), Vol. 5, p. 455.

9. B. A. Hammel, C. Keane, M. Cable, D. Kania, J. Kilkenny, R. Lee, and R. Pasha, Phys. Rev. Lett. 70, 1263 (1993).

10. R. L. Kauffman, L. J. Suter, C. B. Darrow, J. D. Kilkenny, H. N. Komblum, D. S. Montgomery, D. W. Phillion, M. D. Rosen, A. R. Theissen, R. J. Wallace, and F. Ze, Phys. Rev. Lett. 73, 2320 - 2323 (1994).

11. L. J. Suter, R. L. Kauffman, C. B. Darrow, A. A. Hauer, H. N. Kornblum, O. L. Landen, T. J. Orzechowsi, D. W. Phillion, J. L. Porter, L. V. Powers, A. Richard, M. D. Rosen, A. R. Theissen, R. J. Wallace, Phys. Rev. Letters 73, 2328 - 2331 (1994). Phys. Plasmas 3, 2057 - 2062 (1996).

12. E. A. Williams, R. L. Berger, R. P. Drake, A. M. Rubenchik, B. S. Bauer, D. D. Meyerhofer, A. C. Gaeris, and T, W, Johnston, Phys. Plasmas 2, 129 (1995).

13. B. J. MacGowan, B. B. Afeyan, C. A. Back, R. L. Berger, G. Bonnaud, M. Casanova, B. I. Cohen, D. E. Desenne, D. F. Dubois, A. G. Dulieu, K. G. Estabrook, J. C. Fernandez, S. H. Glenzer, D. E. Hinkel, D. H. Kalanter, R. L. Kauffman, R. K. Kirkwood, W. L. Kruer, A. B. Langdon, B. F. Lasinski, D. S. Montgomery, J. D. Moody, D. H. Munro, L. V. Powers, H. A. Rose, C. Rousseaux, R. E. Turner, B. H. Wilde, S. C. Wilks, and E. A. Williams, Phys. Plasmas 3, 2029 - 2040 (1996)

14. R. K. Kirkwood, B. B. Afeyan, C. A. Back, M. A. Blain, D. E. Desenne, K. G. Estabrook, S. H. Glenzer, H. N. Kornblum, B. F. Lasinski, B. J. MacGowan, D. S. Montgomery, J. D. Moody, R. Wallace, and E. A. Williams, Phys. Rev. Lett. 77, 2706 - 2709 (1996).

15. J. C. Fernandez, J. A. Cobble, B. H. Failor, D. F. DuBois, D. S. Montgomery, H. A. Rose, H. X. Vu, B. H. Wilde, M. D. Wilke, and R. E. Chrien, Phys. Rev. Lett. 77, $2702-2705$ (1996).

16. S. H. Glenzer, C. A. Back, K. G. Estabrook, R. Wallace, K. Baker, B. J. MacGowan, B. A. Hammel, R. E. Cid and J. S. De Groot, Phys. Rev. Lett. 77, 1496-1499 (1996)

17. J. A. Fejer, Can. J. Phys. 39, 716 (1961).

18. D. E. Evans, Plasma Phys. 12, 573 (1970).

19. S. H. Glenzer, C. A. Back, L. J. Suter, M. A. Blain, O. L. Landen, J. D. Lindl, B. J. MacGowan, G. F. Stone, R. E. Turner, and B. H. Wilde, Phys. Rev. Lett. (in print)

20. G. B. Zimmerman and W. L. Kruer, Comments Plasma Phys. Controlled Fusion 2, 85 (1975).

21. S. E. Bodner, Comments Plasma Phys. Controlled Fusion 16, 351 (1995).

22. J. A. Stamper and B. H. Ripin, Phys. Rev. Lett. 34, 138 (1975). M. G. Haines, Phys. Rev. Lett. 78, 254 (1997).

23. J. R. Albritton, E. A. Williams, I. B. Bernstein, and K. P. Swartz, Phys. Rev. Letters 57, 1887 (1986). J. F. Luciani, P. Mora, and R. Pellat, Phys. Fluids 28, 835 (1985). A. R. Bell, R. G. Evans, and D. J. Nicholas, Phys. Rev. Lett. 46, 243 (1981).

24. E. M. Campbell, J. T. Hunt, E. S. Bliss, D. R. Speck, and R. P. Drake, Rev. Sci. Intrum. 57, 2101 (1986).

25. E. E. Salpeter, Phys. Rev. 120, 1528 (1960). J. A. Fejer, Can. J. Phys. 38, 1114 (1960). J. P. Dougherty and D. T. Farley, Proc. Roy. Soc. Ser. A 259, 79 (1960). 
26. J. S. De Groot, J. M. Liu, J. P. Matte, D. Perdue, T. W. Johnston, R. P. Drake, K. G. Estabrook, W. L. Kruer, A. B. Langdon, V. Bychenkov, S. Uryupin, and V. Silin, in Laser Interaction and Related Plasma Phenomena (AIP Conf. Proc. No. 318, Plenum Press, 1994), pp. 135 - 143.

27. Th. Wrubel, S. Glenzer, S. Büscher, and H.-J. Kunze, J. Atmos. Terr. Phys. 58, 1077 (1996).

28. A. W. DeSilva, T. J. Baig, I. Olivares, and H.-J. Kunze, Phys. Fluids B 4, 458 (1992).

29. V. T. Tikhonchuk, W. Rozmus, V. Yu. Bychenkov, C. E. Capjack, E. Epperlein, Phys. Plasmas 2, 4169 (1995).

30. L Spitzer, Jr., Phys. Rev. 58, 348 (1940).

31. B. La Fontaine, H. A. Baldis, D. M. Villeneuve, J. Dunn, G. D. Enright, J. C. Kieffer, H. Pepin, M. D. Rosen, D. L. Matthews, and S. Maxon, Phys. Plasmas 1, 2329 (1994).

32 D. S. Hinkel, E. A. Williams, and C. H. Still, Phys. Rev. Lett. 77, 1298 (1996).

33. D. S. Montgomery et al., Phys. Rev. Lett. 73, 2055 (1994). S. H. Batha et al., Phys. Plasmas 2, 3792 (1995). S. H. Glenzer et al., Rev. Sci. Instrum. 68, 668 (1997).

34. B. B. Afeyan, A. E. Chau, and W. L. Kruer, submitted to Phys. Rev. Lett.

35. R. Town, private communication (1997).

36. H. S. Rose, Phys. Plasmas 3, 1709 (1996). J. D. Moody, B. J. MacGowan, D. E. Hinkel, W. L. Kruer, E. A. Williams, K. G. Estabrook, R. L. Berger, R. K. Kirkwood, D. S. Montgomery, and T. D. Shepard, Phys. Rev. Lett. 77, 1294 (1996). 


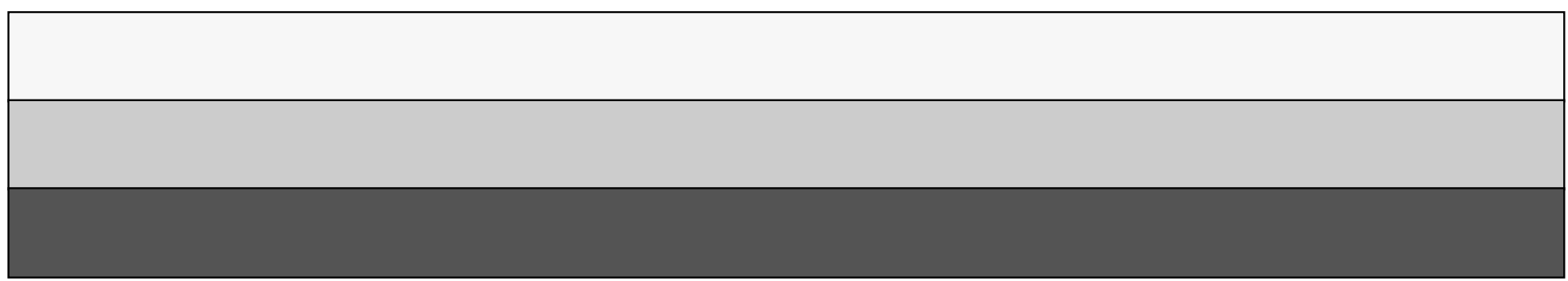

